\title{
Diagnostic value of combination of cranial MRI, serum homocysteine and procalcitonin for hyperbilirubinemia complicated with brain injury in neonates
}

\author{
NA CHANG ${ }^{1,2}$ and GUANGBIN WANG $^{3}$ \\ ${ }^{1}$ Department of Medical Imaging, Shandong University, Jinan, Shandong 250012; \\ ${ }^{2}$ Department of Medical Imaging, Jinan Vocational College of Nursing, Jinan, Shandong 250102; \\ ${ }^{3}$ Shandong Medical Imaging Research Institute, Shandong University, Jinan, Shandong 250021, P.R. China
}

Received June 7, 2019; Accepted April 21, 2020

DOI: 10.3892/etm.2020.9179

\begin{abstract}
The present study aimed to explore the diagnostic value of the combination of cranial magnetic resonance imaging (MRI), serum homocysteine (HCY) and procalcitonin (PCT) for hyperbilirubinemia complicated with brain injury in neonates. One hundred and forty-nine children with hyperbilirubinemia admitted to Shandong Medical Imaging Research Institute from January 2014 to April 2016 were collected as research subjects, and were divided into a brain injury group $(n=67)$ and a non-brain injury group $(n=82)$ according to whether children suffered from brain injury. PCT levels were detected by electrochemiluminescence (ECL), and HCY levels by enzymatic cycling assay (ECA). The combination of cranial MRI, HCY and PCT was used to diagnose hyperbilirubinemia complicated with brain injury in neonates. The concentrations of HCY and PCT in the brain injury group were significantly higher than those in the non-brain injury group $(\mathrm{P}<0.001)$ According to the MRI examination results, the patients were divided into an MRI normal group and an MRI abnormal group. In the brain injury group, the serum HCY and PCT levels of the MRI abnormal group were significantly higher than those of the MRI normal group, with a statistically significant difference $(\mathrm{P}<0.05)$. In the non-brain injury group, the serum HCY and PCT levels of the MRI abnormal group were significantly higher than those of the MRI normal group, with a statistically significant difference $(\mathrm{P}<0.05)$. The sensitivity of the combined detection was significantly higher than that of single detection $(\mathrm{P}<0.05)$; the specificity was significantly higher than that of HCY detection $(\mathrm{P}<0.05)$, and the accuracy
\end{abstract}

Correspondence to: Dr Guangbin Wang, Shandong Medical Imaging Research Institute, Shandong University, 324 Jingwu Road, Jinan, Shandong 250021, P.R. China

E-mail: btqr69@163.com

Key words: cranial MRI, homocysteine, procalcitonin, hyperbilirubinemia complicated with brain injury in neonates, diagnostic value was significantly higher than that of MRI and HCY single detection $(\mathrm{P}<0.05)$. In conclusion, the combination of cranial MRI, HCY and PCT, which has a high diagnostic value for hyperbilirubinemia complicated with brain injury in neonates, is conducive to the early diagnosis and timely treatment of the disease and the reduction of sequelae.

\section{Introduction}

Neonatal hyperbilirubinemia is common, and children with the disease account for approximately $60 \%$ of full-term neonates (1). With complex pathogenesis, it causes an imbalance between the production and elimination of bilirubin $(2,3)$, brain injury, and permanent neurological damage to the subcortical brain structure, even neurological sequelae, such as abnormalities of the vertebral body, hearing impairment and mental retardation (3-5). Brain injury caused by bilirubin is called bilirubin-induced neurologic dysfunction (BIND) (6), which includes acute and chronic bilirubin encephalopathy in neonates (7). Early prediction of brain injury in children with severe hyperbilirubinemia is crucial for improving prognosis. However, it is increasingly difficult to diagnose bilirubin encephalopathy due to its unapparent symptoms in the early stage; therefore, early diagnosis and timely treatment of bilirubin encephalopathy are crucial to reduce the occurrence of sequelae (8). Non-invasive magnetic resonance imaging (MRI) can be clinically used to monitor brain injury caused by hyperbilirubinemia (9).

As the precursor of calcitonin (10), procalcitonin (PCT) is commonly used in the diagnosis of neonatal meningitis, septicemia and bacterial infection (11-13). The concentration of plasma PCT increases and reflects the activity of systemic inflammatory responses in patients with severe fungal, bacterial and parasitic infections and multiple organ failure (14). A study revealed that PCT can be used as a diagnostic indicator for early neonatal septicemia and systemic bacterial infection in children with neonatal hyperbilirubinemia (15). Homocysteine (HCY) is a sulfhydryl-containing amino acid and an intermediate product of methionine and cysteine metabolism, not involved in protein synthesis (16). According to studies, highly expressed HCY was an independent risk 
factor for cardiovascular diseases (17), and its low concentration triggered neuronal injury through oxidative stress, DNA damage and activation of pro-apoptotic factors (18). Currently, there are few studies on the diagnosis of HCY and PCT in neonatal hyperbilirubinemia complicated with brain injury.

Since the early symptoms of hyperbilirubinemia combined with brain injury are not evident, the diagnosis of bilirubin nerve injury is increasingly difficult. Therefore, by studying the MRI examination of the skull and the detection of HCY and PCT, the value of combined diagnosis in predicting brain injury of bilirubin in neonates was evaluated, in order to improve diagnostic performance and enable patients to receive appropriate treatment as soon as possible.

\section{Patients and methods}

Patients. A total of 149 children with hyperbilirubinemia admitted to Shandong Medical Imaging Research Institute from January 2014 to April 2016 were collected as research subjects. The neurological function of the children was assessed based on diagnostic criteria in practical neonatology, and the children were divided into a brain injury group $(n=67)$ and a non-brain injury group $(n=82)$. The brain injury group consisted of 37 males and 30 females, with a gestational age of $(38.8 \pm 1.67)$ weeks, a daily age of $(8.49 \pm 1.49)$ days and a birth weight of $(3575 \pm 73) \mathrm{g}$. The non-brain injury group consisted of 48 males and 34 females, with a gestational age of $(39.1 \pm 1.58)$ weeks, a daily age of $(8.65 \pm 1.54)$ days and a birth weight of $(3561 \pm 75) \mathrm{g}$.

Inclusion criteria were as follows: Diagnostic criteria for bilirubin encephalopathy: i) A serum bilirubin level $\geq 342 \mu \mathrm{mol} / 1$, combining typical nervous system signs and symptoms; ii) abnormal muscle tone; iii) potential abnormalities induced by brainstem auditory dysfunction; iv) brain magnetic resonance imaging exhibiting bilateral globus pallidus signal abnormalities; v) children with complete clinical data; children with normal liver, spleen and other organs in size; children who were fed with breast milk; children who had not received albumin, gamma globulin and phototherapy; children without a history of intrauterine distress and asphyxia; children without congenital malformation.

Exclusion criteria: Children with severe dysfunction of the heart, kidney and liver; children with nervous system diseases caused by heredity or metabolism; children with other severe brain diseases.

The present study was approved by the Ethics Committee of Shandong Medical Imaging Research Institute. The family members of the subjects were informed and signed a complete informed consent form.

Collection and detection of serum. Fasting venous blood ( $2 \mathrm{ml}$ ) was collected within $24 \mathrm{~h}$ of admission and centrifuged at $4^{\circ} \mathrm{C}$ and $1,000.46 \times \mathrm{g}$ for $10 \mathrm{~min}$ to collect the supernatant, which was placed at $-80^{\circ} \mathrm{C}$ for testing and prevented from repeated freezing and thawing. PCT was detected by electrochemiluminescence (ECL) with a Roche E601 automatic ECL analyzer and Roche reagents (Procell and Cleancell). HCY was detected by enzymatic cycling assay (ECA) (19) with a Mindray BS-220 automatic biochemical analyzer and HCY kit (cat. no. 181001) provided by Zybio, Inc.
Cranial MRI. All children were induced to fall asleep 15-20 min before examination, and administered oral 10\% chloral hydrate $(30-40 \mathrm{mg} / \mathrm{kg})$ or $10 \%$ chloral hydrate enema. During scanning, sound-proof cotton was placed in the external auditory canal of the children, and plastic sponge mats were used to fix the heads of the children, so that the position line was aligned with the radiographic base line. The children were examined using the Phillip Achieve 1.5 T double gradient superconducting MRI system and an 8-channel phased array head coil. Parameters of T1-weighted imaging (T1WI): Spin echo (SE) sequence, TR=450 ms, TE=10 ms. Parameters of T2-weighted imaging (T2WI): TR=4,600 ms, TE=110 ms, layer thickness $=5 \mathrm{~mm}$, interlayer spacing $=1.5 \mathrm{~mm}$, imaging matrix $=220 \times 256 \mathrm{~mm}$, imaging layers $=15$. Parameters of diffusion-weighted imaging (DWI): Echo planar imaging (EPI), diffusion factor $b=1,000 \mathrm{~s} / \mathrm{mm}^{2}$. Volumetric data were transmitted to the workstation, and images were analyzed using the AW4.6/FuncTool software (General Healthcare). All image data were read by two senior radiologists using the single-blind method, then analyzed and summarized based on the results. The high-intensity signals in the posterior part of the globus pallidus with $\mathrm{T} 1$ and $\mathrm{T} 2$ weighting were positive (20). All subjects underwent MRI, and they were classified into an MRI abnormal group and an MRI normal group according to the positive signs.

Observational indexes. The expression levels of HCY and PCT in the two groups were detected and compared. Based on the receiver operating characteristic (ROC) curve, the maximum Youden index was determined to obtain the sensitivity and specificity, and the corresponding data were determined according to the ROC. Then the optimal cut-off values of HCY and PCT were determined. Expression higher than the optimal cut-off value indicated positive, while expression lower than that indicated negative. The sensitivity, specificity and accuracy of the combined detection of cranial MRI, HCY and PCT were investigated.

Statistical analysis. SPSS 19.0 (IBM Corp.) was used to statistically analyze the data. Count data were expressed by [n(\%)], and chi-square test was used for comparisons between groups. Measurement data were expressed by (mean $\pm \mathrm{SD}$ ), and t-test was used for comparisons between two groups. ROC was used to evaluate the diagnostic value of HCY and PCT. $\left.\mathrm{Z}=|\mathrm{AUC} 1-\mathrm{AUC} 2| / \operatorname{sqrt}_{(\mathrm{SE} 1}{ }^{\wedge} 2+\mathrm{SE}^{\wedge} 2\right)$ was used to calculate $\mathrm{Z}$ values, and then the $\mathrm{P}$-value was calculated according to the normal distribution. $\mathrm{P}<0.05$ indicated a statistically significant difference.

\section{Results}

Comparison of general information. There was no statistically significant difference between the brain and non-brain injury groups in terms of sex, gestational age, daily age, average birth weight, means of pregnancy, head circumference, the age of the mother or average gestational week $(\mathrm{P}>0.05$; Table I).

Comparison of the expression levels of HCY and PCT. According to the results (Figs. 1 and 2), the expression levels of $\mathrm{HCY}$ and PCT in the brain injury group were $27.56 \pm 6.26 \mu \mathrm{mol} / 1$ 
Table I. Comparison of the general information $[\mathrm{n}(\%)] /($ mean $\pm \mathrm{SD})$.

\begin{tabular}{|c|c|c|c|c|}
\hline Variable & Brain injury group $(n=67)$ & Non-brain injury group $(n=82)$ & $\chi^{2} /$ t-test & P-value \\
\hline Sex & & & 0.165 & 0.685 \\
\hline Male & $37(55.2)$ & $48(58.5)$ & & \\
\hline Female & $30(44.8)$ & $34(41.5)$ & & \\
\hline Gestational age (weeks) & $38.80 \pm 1.67$ & $39.10 \pm 1.58$ & 1.124 & 0.263 \\
\hline Daily age (days) & $8.49 \pm 1.49$ & $8.65 \pm 1.54$ & 0.640 & 0.523 \\
\hline Birth weight (g) & $3575 \pm 73$ & $3561 \pm 75$ & 1.147 & 0.253 \\
\hline Means of pregnancy & & & 0.020 & 0.889 \\
\hline Eutocia & $36(53.7)$ & $45(54.9)$ & & \\
\hline Cesarean delivery & $31(46.3)$ & $37(45.1)$ & & \\
\hline Head circumference $(\mathrm{cm})$ & $32.87 \pm 0.57$ & $33.02 \pm 0.59$ & 1.567 & 0.119 \\
\hline Age of the mother (years) & $29.12 \pm 2.89$ & $28.76 \pm 2.97$ & 0.745 & 0.458 \\
\hline Average gestational weeks & $39.12 \pm 1.38$ & $38.79 \pm 1.41$ & 1.435 & 0.154 \\
\hline
\end{tabular}

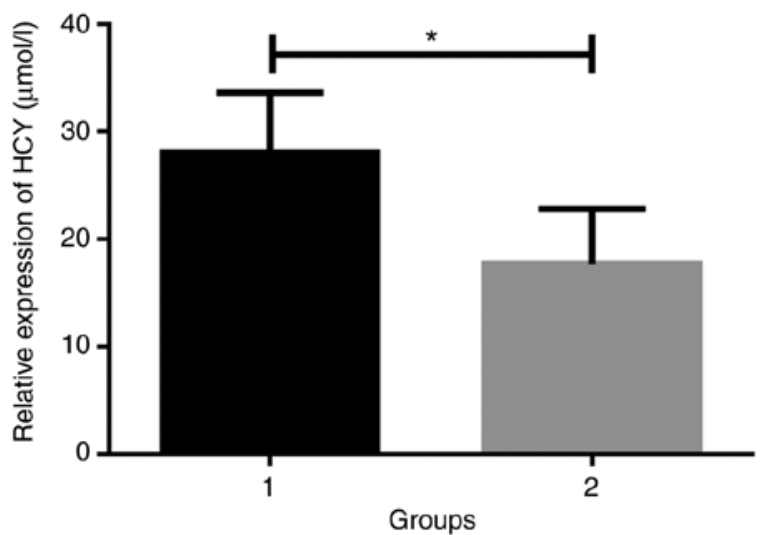

Figure 1. Comparison of the expression level of HCY. According to the ECA, the expression of HCY in the brain injury group was $27.56 \pm 6.26 \mu \mathrm{mol} / 1$, significantly higher than $17.26 \pm 5.21 \mu \mathrm{mol} / 1$ in the non-brain injury group $(\mathrm{t}=10.96, \mathrm{P}<0.001)$. The brain injury group is indicated by the number 1 in the image, and the non-brain injury group by 2 . ${ }^{*} \mathrm{P}<0.05$ compared with the brain injury group. $\mathrm{HCY}$, homocysteine; ECA, enzymatic cycling assay.

and $1.57 \pm 1.29 \mathrm{ng} / \mathrm{ml}$, and those in the non-brain injury group were $17.26 \pm 5.21 \mu \mathrm{mol} / 1$ and $0.34 \pm 0.31 \mathrm{ng} / \mathrm{ml}$. The concentrations of HCY and PCT in the brain injury group were significantly higher than those in the non-brain injury group $(\mathrm{t}=11.75$ and $9.41, \mathrm{P}<0.001)$.

Analysis of images of MRI. Representative images of MRI are presented in Fig. 3. The different images were obtained from one patient. In the acute phase of neonatal bilirubin encephalopathy, the MRI scan revealed increased symmetry of T1 signal in the bilateral globus pallidus and internal capsule; T2 showed equal signal; DWI sequence showed equal signal; the ventricular system exhibited no expansion; there was no broadening and deepening of cerebral sulcus, and the centerline structure was centered.

The MRI images of normal newborns can be observed in Fig. 4. The DWI, the T1 weighted, the T2-FLAIR and the T2 weighted sequences are presented. As indicated by the arrows, the signals of the pale ball on both sides of the T1 weighted

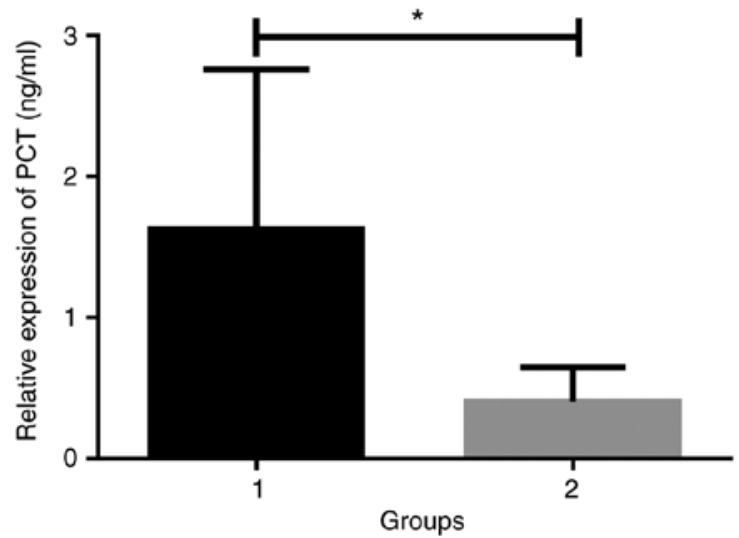

Figure 2. Comparison of expression level of PCT. According to ECL, the expression of PCT in the brain injury group was $1.57 \pm 1.29 \mathrm{ng} / \mathrm{ml}$, significantly higher than $0.34 \pm 0.31 \mathrm{ng} / \mathrm{ml}$ in the non-brain injury group $(\mathrm{t}=8.35$, $\mathrm{P}<0.001)$. The brain injury group is indicated by the number 1 in the image, and the non-brain injury group by $2 .{ }^{*} \mathrm{P}<0.05$ compared with the brain injury group. PCT, procalcitonin; ECL, electrochemiluminescence.

sequence increased, and no obvious abnormal signals were observed in the T2 weighted and T2-FLAIR sequences.

Relationship between MRI results and HCY and PCT levels in serum. According to the MRI examination results, the patients were divided into an MRI normal group and an MRI abnormal group. There was no significant difference in the general characteristics between the two groups ( $\mathrm{P}>0.05$; Table II). In the brain injury group, the serum HCY and PCT levels of the MRI abnormal group were significantly higher than those of the MRI normal group, with a statistically significant difference $(\mathrm{P}<0.05)$. In the non-brain injury group, the serum HCY and PCT levels of the MRI abnormal group were significantly higher than those of the MRI normal group, with a statistically significant difference $(\mathrm{P}<0.05$; Table III).

Diagnostic values of MRI, HCY and PCT. According to ROC curve, the optimal cutoff values of HCY and PCT were respectively 19.78 and 0.89 (Fig. 5). Altogether 65 cases 
Table II. Comparison of the general information between the MRI normal group and the MRI abnormal group [n(\%)]/(mean $\pm \mathrm{SD})$.

\begin{tabular}{|c|c|c|c|c|}
\hline Variable & MRI normal group $(n=84)$ & MRI abnormal group $(n=65)$ & $\chi^{2} /$ t-test & P-value \\
\hline Sex & & & 0.000 & 0.980 \\
\hline Male & $48(57.14)$ & $37(56.92)$ & & \\
\hline Female & $36(42.86)$ & $28(43.08)$ & & \\
\hline Gestational age (weeks) & $39.11 \pm 1.57$ & $38.86 \pm 1.60$ & 0.960 & 0.341 \\
\hline Daily age (days) & $8.61 \pm 1.51$ & $8.39 \pm 1.55$ & 0.872 & 0.385 \\
\hline Birth weight (g) & $3571 \pm 78$ & $3547 \pm 81$ & 1.832 & 0.069 \\
\hline Means of pregnancy & & & 0.196 & 0.658 \\
\hline Eutocia & $47(55.95)$ & $34(52.31)$ & & \\
\hline Cesarean delivery & $37(44.05)$ & $31(47.69)$ & & \\
\hline Head circumference $(\mathrm{cm})$ & $32.88 \pm 0.61$ & $33.01 \pm 0.57$ & 1.327 & 0.187 \\
\hline Age of the mother (years) & $28.25 \pm 2.63$ & $29.04 \pm 2.77$ & 1.777 & 0.078 \\
\hline Average gestational weeks & $39.09 \pm 1.33$ & $38.69 \pm 1.38$ & 1.791 & 0.075 \\
\hline
\end{tabular}

MRI, magnetic resonance imaging.
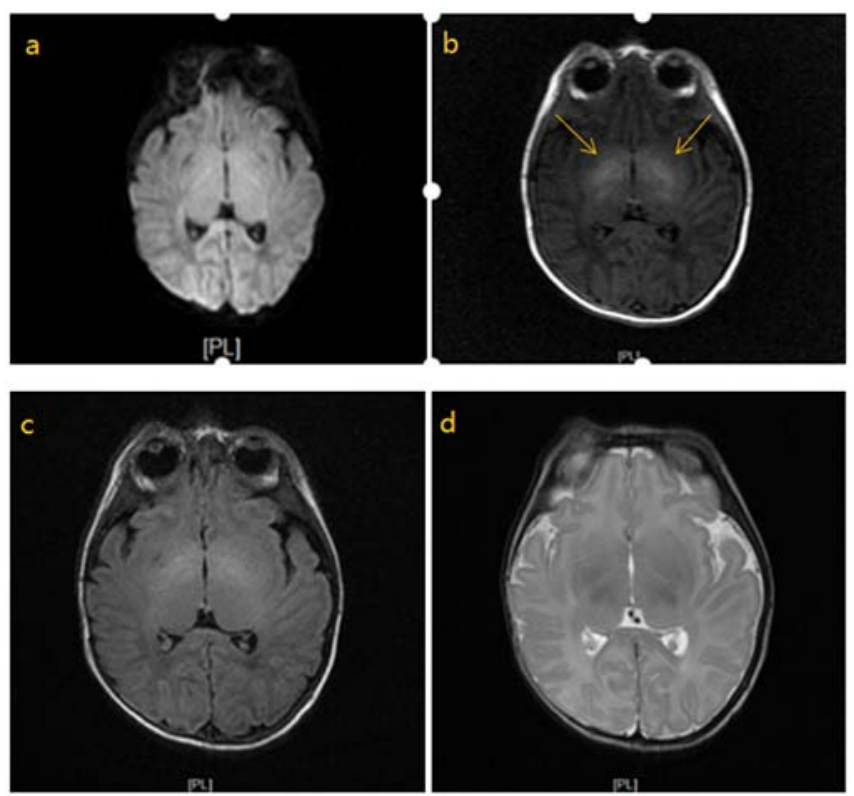

Figure 3. Representative images of MRI in neonatal hyperbilirubinemia. (a) DWI sequence; (b) T1-weighted sequence; (c) T2-FLAIR sequence; and (d) T2-weighted sequence. There was an increase in bilateral pterygium pallidus signals in the T1-weighted sequence (as indicated by the arrows), a slight increase in T2-flair signals, and no obvious abnormal signals in the DWI and T2-weighted sequences. MRI, magnetic, resonance imaging; DWI, diffusion-weighted imaging.

of hyperbilirubinemia complicated with brain injury were detected by MRI, including 52 cases of true positive and 69 cases of true negative. Altogether 79 cases were detected by HCY, including 53 of true positive and 56 of true negative. Altogether 46 cases were detected by PCT, including 43 of true positive and 79 of true negative. Altogether 67 cases were detected by the combination of MRI, HCY and PCT, including 60 of true positive and 75 of true negative (Table IV). Comparison of the diagnostic value between single and combined detection is presented in Table V. The sensitivity of the combined detection was significantly higher than that
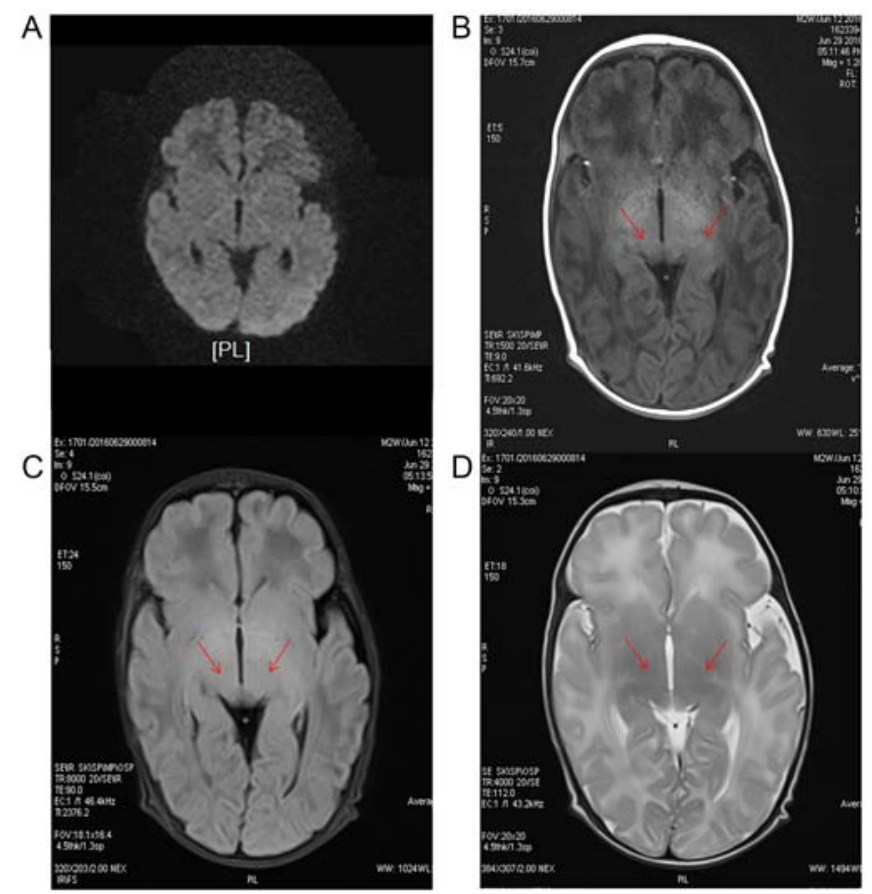

Figure 4.MRI images of normal newborns.(A) DWI sequence; (B) T1-weighted sequence; (C) T2-FLAIR sequence and (D) T2-weighted sequence. At the arrows, the signals of the pale ball on both sides of the T1-weighted sequence was increased, and no obvious abnormal signals were observed in the T2-weighted sequence and T2-FLAIR sequence. MRI, magnetic, resonance imaging; DWI, diffusion-weighted imaging.

of single detection $(\mathrm{P}<0.05)$, the specificity was significantly higher than that of HCY detection $(\mathrm{P}<0.05)$, and the accuracy was significantly higher than that of MRI and HCY single detection $(\mathrm{P}<0.05)$.

\section{Discussion}

In recent years, neonatal hyperbilirubinemia and its bilirubin brain damage have drawn considerable attention. Excessive 
Table III. Association between MRI results and serum HCY and PCT levels (mean $\pm \mathrm{SD}$ ).

\begin{tabular}{lccc}
\hline Groups & Cases & $\mathrm{HCY}(\mu \mathrm{mol} / \mathrm{l})$ & $\mathrm{PCT}(\mathrm{ng} / \mathrm{ml})$ \\
\hline Brain injury group & & & \\
MRI abnormal group & 52 & $29.27 \pm 9.01$ & $1.86 \pm 1.45$ \\
MRI normal group & 15 & $17.88 \pm 7.87$ & $0.37 \pm 0.29$ \\
t-value & & 4.995 & 5.590 \\
P-value & & $<0.001$ & $<0.001$ \\
Non-brain injury group & & & \\
MRI abnormal group & 13 & $25.09 \pm 6.78$ & $1.32 \pm 0.87$ \\
MRI normal group & 69 & $14.12 \pm 6.47$ & $0.28 \pm 0.19$ \\
t-value & & 4.663 & 3.782 \\
P-value & & $<0.001$ & $<0.001$ \\
\hline
\end{tabular}

MRI, magnetic resonance imaging; HCY, homocysteine; PCT, procalcitonin.

Table IV. Result statistics on single and combined detection.

\begin{tabular}{lrrr}
\hline Cases & + & - & Sum \\
\hline MRI & & & \\
+ & 52 & 13 & 65 \\
- & 15 & 69 & 84 \\
Sum & 67 & 82 & 149 \\
HCY & & & \\
+ & 53 & 26 & 79 \\
- & 14 & 56 & 70 \\
Sum & 67 & 82 & 149 \\
PCT & & & \\
+ & 43 & 3 & 103 \\
- & 24 & 79 & 149 \\
Sum & 67 & 82 & \\
MRI+HCY+PCT & & & 67 \\
+ & 60 & 7 & 149 \\
- & 7 & 75 & \\
Sum & 67 & 82 & \\
\hline
\end{tabular}

MRI, magnetic resonance imaging; HCY, homocysteine; PCT, procalcitonin.

free bilirubin passes through the blood-cerebrospinal fluid barrier and deposits in the brain tissue, resulting in bilirubin encephalopathy, which is a brain tissue injury leading to death if patients are not treated timely $(21,22)$. Therefore, early diagnosis and timely treatment are crucial for reducing brain tissue injury and controlling disease progression (23). MRI which is non-invasive, non-radiative and atraumatic, is an imaging technique that uses magnetic field signals generated by hydrogen protons in human biological tissues to image (24). According to a study on the analysis of MRI signal characteristics of severe neonatal hyperbilirubinemia, T1WI images with STN/T and GP/T ratios greater than 1.63 and 1.56
Table V. Comparison of the diagnostic value between single and combined detection.

\begin{tabular}{lccc}
\hline Indicators & Sensitivity $\%$ & Specificity $\%$ & Accuracy $\%$ \\
\hline MRI & $77.6^{\mathrm{a}}$ & 84.1 & $81.2^{\mathrm{a}}$ \\
HCY & $79.1^{\mathrm{a}}$ & $68.3^{\mathrm{a}}$ & $73.2^{\mathrm{a}}$ \\
PCT & $64.2^{\mathrm{a}}$ & 96.3 & 81.9 \\
HCY+PCT & $73.13^{\mathrm{a}}$ & 92.68 & 83.89 \\
MRI+HCY+PCT & 90.0 & 91.5 & 90.6 \\
\hline
\end{tabular}

${ }^{\mathrm{a}} \mathrm{P}<0.05$ when compared with the combined detection. MRI, magnetic resonance imaging; HCY, homocysteine; PCT, procalcitonin.

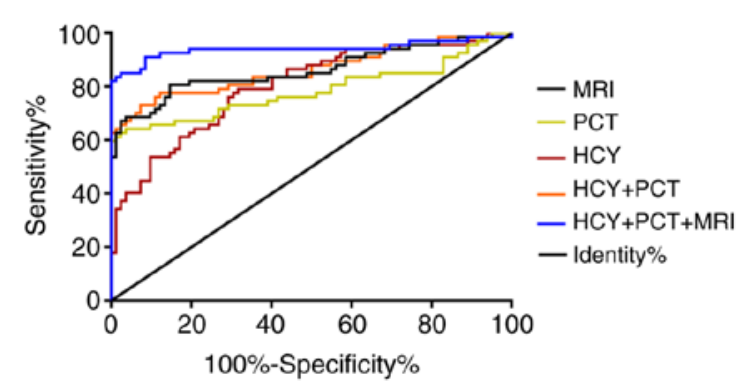

Figure 5. Diagnostic value of serum HCY and PCT in neonatal hyperbilirubinemia brain injury. According to the ROC curve, the AUC of HCY was 0.800 , and the optimal cut-off value was 19.78. The AUC of PCT was 0.785 , and the optimal cut-off value was 0.89 . HCY, homocysteine; PCT, procalcitonin; ROC, receiver operating characteristic; AUC, area under the curve; MRI, magnetic, resonance imaging.

indicated bilirubin encephalopathy, and poor prognosis usually manifested as a symmetrical high signal on T2WI at the same position in chronic phase (25). Therefore, early diagnosis of neonatal hyperbilirubinemia complicated with brain injury is of great significance.

High concentration of HCY induces the increase of $\mathrm{S}$-adenosyl homocysteine $(\mathrm{SAH})$ which reduces the ratio of S-adenosyl methionine (SAM) to SAH, and the protein expression and enzyme activity of DNA methyltransferase, thus reducing DNA methylation levels, inhibiting the proliferation of neural stem cells and resulting in systemic diseases through molecular mechanisms of the negative regulation (26). PCT, whose expression level is very low in healthy people, is a glycoprotein without hormone activity and produced by thyroid $\mathrm{C}$ cells (27). A study revealed that PCT was slightly increased in viral infections but significantly increased in bacterial infections, so it is helpful to distinguish bacterial meningitis from viral meningitis (28).

In the present study, the concentrations of HCY and PCT in the brain injury group were significantly higher than those in the non-brain injury group, indicating that HCY and PCT are highly expressed in children with brain injury. According to studies, HCY is highly expressed in diseases $(29,30)$, and its high expression is a risk factor for neonatal hyperbilirubinemia (31). In a study on hyperbilirubinemia, the expression level of PCT in the infection group was significantly higher than that in the non-infection group, and the combined detection 
had a high diagnostic value for infection and non-infection in neonatal hyperbilirubinemia (14). It was therefore suggested that HCY and PCT were involved in the occurrence and progression of hyperbilirubinemia, and have a high diagnostic value in the complications of the disease. A study revealed that bilateral globus pallidus of neonatal bilirubin encephalopathy was significant, and its symmetrical high signal was an important feature of MRI (24). This is similar to the results of the present study. In a study by Zhang et al on children with severe hyperbilirubinemia (32), the NBNA score in the abnormal MRI group was lower than that in the normal MRI group. In a study on children with hyperbilirubinemia, MRI was correlated with serum liver function indexes (33). Therefore, MRI may be correlated with serum markers of neonatal hyperbilirubinemia. The specific correlation remains to be explored in further research.

In the present study, MRI findings of bilirubin encephalopathy patients revealed increased symmetry of T1 signals in bilateral globus pallidus and internal sacs, equal signals in T2, equal signals in DWI sequence, no expansion of ventricular system, no widening or deepening of sulci and fissure, and the centerline structure was centered. In the present study, there were a few positive MRI signals in the non-brain injury group, which was mainly considered as the high positive rate in the bilirubin encephalopathy group, suggesting that the high intensity signal in the posterior part of the MRI T1 and T2 weighted image of the blebus was the most characteristic change in bilirubin brain injury. In the non-brain injury group, positive signals may exist due to the severity of the disease and abnormalities, but the positive rate is low $(20,34,35)$. It has been reported that serum HCY has high sensitivity and specificity in vascular mild cognitive dysfunction in patients with cerebral small vessel disease, and can be used as a predictor of vascular mild cognitive dysfunction in patients with cerebrovascular disease (36). PCT can be used as a rapid and accurate reference for early diagnosis of severe brain injury complicated with pulmonary infection (37), and serum PCT was a more sensitive indicator for predicting the risk of multiple organ dysfunction syndrome or death in patients with brain injury (38). In the present study, according to ROC, HCY and PCT can be used as diagnostic indicators for the diagnosis of neonatal hyperbilirubinemia brain injury. The optimal cutoff value was calculated. The sensitivity of MRI combined with serum HCY and PCT was significantly higher than that of single detection, and the specificity was significantly higher than that of HCY detection, and the accuracy was significantly higher than that of MRI and HCY single detection $(\mathrm{P}<0.05)$. A study revealed that NBNA score combined with cranial MRI had a high diagnostic value for severe neonatal hyperbilirubinemia complicated with brain injury (32). According to Li et al (39), the combined detection of MRI, caspase-1 and interleukin- 6 had a high diagnostic value for white matter injury in premature infants. Therefore, MRI combined with serum markers can significantly improve the diagnostic rate of brain diseases.

There are currently few comparative studies on the sensitivity and specificity of cranial MRI combined with HCY and PCT in detecting neonatal hyperbilirubinemia complicated with brain injury. The diagnostic value of the combined detection was comprehensively explored in this study, however there are still limitations. Children were not followed-up, and there was no data on their long-term prognosis. Therefore, a multi-center and systematic study with large samples is required to improve the application value for the diagnosis and treatment of brain injury.

In summary, the combination of cranial MRI, HCY and PCT, which has a high diagnostic value for neonatal hyperbilirubinemia complicated with brain injury, was conducive to the early diagnosis and timely treatment of the disease and the reduction of sequelae.

\section{Acknowledgements}

Not applicable.

\section{Funding}

No funding was received.

\section{Availability of data and materials}

The datasets used and/or analyzed during the current study are available from the corresponding author on reasonable request.

\section{Authors' contributions}

NC performed ECL, and wrote the manuscript. GW was responsible for ECA, and revised the manuscript critically for important intellectual content. $\mathrm{NC}$ and GW analyzed and interpreted the data of the patients, and processed the statistics. Both authors read and approved the final manuscript.

\section{Ethics approval and consent to participate}

The study was approved by the Ethics Committee of Shandong Medical Imaging Research Institute. The family members of the patients who participated in this research, signed the informed consent and all patients had complete clinical data.

\section{Patient consent for publication}

Not applicable.

\section{Competing interests}

The authors declare that they have no competing interests.

\section{References}

1. Zheng J, Wei C, Zhao M and Zhao D: Phototherapy is associated with the decrease in serum globulin levels in neonatal hyperbilirubinemia. Biomed Rep 10: 63-69, 2018.

2. Yu ZB, Han SP and Chen C: Bilirubin nomograms for identification of neonatal hyperbilirubinemia in healthy term and late-preterm infants: A systematic review and meta-analysis. World J Pediatr 10: 211-218, 2014.

3. Petersen JP, Henriksen TB, Hollegaard MV, Vandborg PK, Hougaard DM, Thorlacius-Ussing O and Ebbesen F: Extreme neonatal hyperbilirubinemia and a specific genotype: A population-based case-control study. Pediatrics 134: 510-515, 2014.

4. Kuzniewicz MW, Wickremasinghe AC, Wu YW, McCulloch CE, Walsh EM, Wi S and Newman TB: Incidence, etiology, and outcomes of hazardous hyperbilirubinemia in newborns. Pediatrics 134: 504-509, 2014. 
5. Koziol LF, Budding DE and Chidekel D: Hyperbilirubinemia: Subcortical mechanisms of cognitive and behavioral dysfunction. Pediatr Neurol 48: 3-13, 2013.

6. Smitherman H, Stark AR and Bhutani VK: Early recognition of neonatal hyperbilirubinemia and its emergent management. Semin Fetal Neonatal Med 11: 214-225, 2006.

7. Maisels MJ: Neonatl jaundice. Pediatr Rev 27: 443-454, 2006.

8. Qian-Qian F, Shao-Hua W and Department P: The clinical progress in early diagnosis of bilirubin encephalopathy. Med Recap 20: 1186-1189, 2014.

9. Shi-Xin FU, Kai-Zhong Z, Yan B, et al: Imaging features and clinical outcome of MRI and MRS in children with bilirubin encephalopathy. Chin J CT MRI 16: 11-13, 2018.

10. Monsef A and Eghbalian F: Evaluation of diagnostic value of procalcitonin as a marker of neonatal bacterial infections. Iran J Pediatr 22: 314-318, 2012

11. Czyzewska M, Lachowska M and Gajewska E: Evaluation of diagnostic value of procalcitonin (PCT) as a marker of congenital infection in newborns. Przegl Lek 59 (Suppl 1): S46-S49, 2002 (In Polish).

12. Kawezymksi P and Piotrowski A: Procalcitonin and C-reactive protein as a markers of neonatal sepsis. Cinekol Pol 75: 439-444, 2004 (In Polish).

13. Zahedpasha Y, Ahmadpour M, Hajiahmadi M and Haghshenas M: Procalcitonin as a marker of neonatal sepsis. Iran J Pediatr 19: 117-122, 2009.

14. Weidong HE and Laboratory DO: Application value of immature granulocyte, D-dimer, FDP and PCT detection in neonatal hyperbilirubinemia. China Health Stand Manage 7: 145-147, 2016.

15. Bo G, Feng L, Guoxin X, et al: Clinical significance of procalcitonin, C-reactive protein and myocardial enzymes determination in patients with neonatal hyperbilirubinemia. Int J Lab Med 35: 2441-2443, 2014

16. Joshi MB, Baipadithaya G, Balakrishnan A, Hegde M, Vohra M, Ahamed R, Nagri SK, Ramachandra L and Satyamoorthy K: Elevated homocysteine levels in type 2 diabetes induce constitutive neutrophil extracellular traps. Sci Rep 6: 36362, 2016.

17. Mangge H, Becker K, Fuchs D and Gostner JM: Antioxidants, inflammation and cardiovascular disease. World J Cardiol 6: 462-477, 2014

18. Curro M, Gugliandolo A, Gangemi C, Risitano R, Ientile R and Caccamo D: Toxic effects of mildly elevated homocysteine concentrations in neuronal-like cells. Neurochem Res 39: $1485-1495,2014$.

19. Wu F, Yang H and Liu B: Association between homocysteine and arterial stiffness in women with a history of preeclampsia. J Vasc Res 56: 152-159, 2019.

20. Dan-Ni YE, Ling-Qing GE and Sheng-Lin YU: Diagnostic significance of brainstem auditory evoked potential combined with MRI in early neonatalbilirubin encephalopathy. Chin J Child Health Care 25: 737-740, 2017.

21. Yan B, Kaizhong Z, Qian Z, et al: Diagnostic performance compared of MRI and MRS in neonatal acute bilirubin encephalopathy. Chin Imaging J Integr Tradit West Med, 2017.

22. Sgro M, Campbell D, Barozzino T and Shah V: Acute neurological findings in a national cohort of neonates with severe neonatal hyperbilirubinemia. J Perinatol 31: 392-396, 2011.

23. Iskander I, Gamaleldin R, El Houchi S, El Shenawy A, Seoud I, El Gharbawi N, Abou-Youssef H, Aravkin A and Wennberg RP: Serum bilirubin and bilirubin/albumin ratio as predictors of bilirubin encephalopathy. Pediatrics 134: e1330-e1339, 2014.

24. Wisnowski JL, Panigrahy A, Painter MJ and Watchko JF: Magnetic resonance imaging of bilirubin encephalopathy: current limitations and future promise. Semin Perinatol 38: 422-428, 2014.
25. Xiao-Li M, Lei Z, Xiao-Hu W, et al: Analysis of MRI signal characteristics of neonatal severe hyperbilirubinemia. Chin J Magn Reson Imaging 9: 768-772, 2018.

26. Lin N, Qin S, Luo S, Cui S, Huang G and Zhang X: Homocysteine induces cytotoxicity and proliferation inhibition in neural stem cells via DNA methylation in vitro. FEBS J 281: 2088-2096, 2014.

27. Peschanski N, Chenevier-Gobeaux C, Mzabi L, Lucas R, Ouahabi S, Aquilina V, Brunel V, Lefevre G and Ray P: Prognostic value of PCT in septic emergency patients. Ann Intensive Care 6: 47, 2016.

28. Velissaris D, Pintea M, Pantzaris N, Spatha E, Karamouzos V, Pierrakos $\mathrm{C}$ and Karanikolas M: The role of procalcitonin in the diagnosis of meningitis: A literature review. J Clin Med 7: 148, 2018.

29. La'ulu SL, Rawlins ML, Pfeiffer CM, Zhang M and Roberts WL: Performance characteristics of six homocysteine assays. Am J Clin Pathol 130: 969-975, 2008.

30. Moretti R and Caruso P: The controversial role of homocysteine in neurology: From labs to clinical practice. Int J Mol Sci 20: 231, 2019.

31. Sukla KK, Tiwari PK, Kumar A and Raman R: Low birthweight (LBW) and neonatal hyperbilirubinemia $(\mathrm{NNH})$ in an Indian cohort: Association of homocysteine, its metabolic pathway genes and micronutrients as risk factors. PLoS One 8: e71587, 2013.

32. Zhang HY, Qiao LX, Zhu WY and Wang Hua:: Diagnostic value of NBNA score combined head MRI in the neonates with severe hyperbilirubinemia for brain injury. Chin J Child Health Care 25: 164-166, 2017.

33. Yanming GE, Yaowu LI and Qinyan XU: The study on the correlation between features of magnetic resonance imaging and serum index about hyperbilirubinemia of the neonates. J Clin Radiol 34: 1468-1471, 2015.

34. Chen LJ, Wang XM, Wan YZ, Li WH, Jia YG, Yun CH and Chen TH: Superconducting MRI signal intensity in pallidum in neonatal hyperbilirubinemia. Chin J Rehabil Theory Pract 22: 838-840, 2016 (In Chinese)

35. Li XH, Zhang J, Zheng H, et al: Clinical significance of cerebrospinal fluid bilirubin and craniocerebral magnetic resonance imaging measurements in newborns with bilirubin encephalopathy. Pract Clin Med 4: 76-79, 2014.

36. Wang T, Sun ZW, Shao LQ, Xu XB, Liu Y, Qin M, Weng X and Zhang YX: Diagnostic values of serum levels of homocysteine and uric acid for predicting vascular mild cognitive impairment in patients with cerebral small vessel disease. Med Sci Monit 23: 2217-2225, 2017.

37. Wei W, Jinyue C, Qing Z, Chunxiao F, Min L and Mingfen L: Early diagnosis value of procalcitonin in severe brain damage combined with pulmonary infection. Int J Lab Med: 2934-2936, 2015 (In Chinese)

38. Ai-Hua F, Shu-Ming P, Ming L, et al: Value of procalcitonin to the prediction of multiple organ dysfunction syndrome in patients with brain injury. J Chin Pract Diagn Ther, 2011.

39. Li Y: Application of MRI combined with caspase- 1 and IL-6 in the diagnosis of white matter damage in premature infants. Lab Med Clin 14: 2211-2213, 2017.

This work is licensed under a Creative Commons Attribution-NonCommercial-NoDerivatives 4.0 International (CC BY-NC-ND 4.0) License. 